\title{
Demagnetizing the drill string magnetic interference in Far North and in Pakistan
}

\author{
Shaine Mohammadali Lalji ${ }^{1,2} \cdot$ Javed Haneef ${ }^{1,2} \cdot$ Muhammad Arqam Khan ${ }^{1,2} \cdot$ Syed Imran Ali $^{1,2}$
}

Received: 6 March 2019 / Accepted: 4 November 2019 / Published online: 16 November 2019

(c) The Author(s) 2019

\begin{abstract}
Drilling in Barents Sea proves to be a challenging task, as this region is situated in auroral zones having high geomagnetic latitude, where magnetic interferences develop from magnetic field and magnetic materials inside subsurface are quite common. For this region, monitoring of magnetic field is utterly significant as any fluctuations can distort the tool sensor performance with ultimately enlarging the uncertainty in azimuth. To guide a well to its desire location, measurement while drilling (MWD) tool needs to be operated with utmost precision; however, its accuracy compromises as a result of magnetic interferences from drill string and nearby magnetic material. The performance of this tool depends upon its sensors. Any distortion in sensor performance can lead to problems such as multiple sidetracking and increase in overall project cost. Furthermore, the same BHA was also placed in a region of Pakistan and the impact drill string interference was observed. It was discovered that the interferences that had tremendous impact on magnetometer $Z$-component in Barents Sea had a drastic reduction in the region of Pakistan as it is situated in low latitude, where uncertainty in azimuth is low. In this work, an exemplary bottom-hole assembly (BHA) was analyzed and the impact of individual drill string components interferences was observed on the MWD sensors. It was perceived that the bit was responsible for creating the major distortion in MWD sensor. Apart from that, it was also investigated that the location of the well also plays a vital role in this distortion. This intervention in the sensors is created by a vast difference between the used actual length and the recommended length of nonmagnetic drill collar in the BHA. Numerically, it was investigated that if the physical distance between the sensors and bit is increased, then this interference is reduced. It was also apparent that the $Z$-component of the magnetometer was utterly distorted because of this interference, while the $X$ - and $Y$-components were proved to be independent of these interferences. It was further examined that the effects of latitude and longitude play a significant role in the course of changing the impact of these errors on magnetization.
\end{abstract}

Keywords Magnetic interference $\cdot$ Measurement while drilling $\cdot$ Bottom-hole assembly $\cdot$ Sensors $\cdot$ Nonmagnetic drill collars (NMDC)

Abbreviations

MWD Measurement while drilling

NMDC Nonmagnetic drill collar

Shaine Mohammadali Lalji

shaine@neduet.edu.pk

Javed Haneef

javedh@neduet.edu.pk

Muhammad Arqam Khan

arqamkhan@neduet.edu.pk

Syed Imran Ali

engrimran@neduet.edu.pk

1 Department of Petroleum Engineering, NED University of Engineering and Technology, Karachi, Pakistan

2 Pakistan Engineering Council (PEC), Islamabad, Pakistan
BHA Bottom-hole assembly

DS Drill string

UBHO Universal Bottom Hole Orientation Subs

TMF Total magnetic field

MB Magnetometer bias

WMM World magnetic model

\section{List of symbols}

$H_{a d} \quad$ Applied magnetic field

$\mu_{o} \quad$ Magnetic permeability of free space

$N \quad$ Demagnetizing factor

$\mu_{\max } \quad$ Maximum permeability of BHA component ( $\mathrm{Wb} \mathrm{m} / \mathrm{A}$ )

$\mu_{\text {min }} \quad$ Minimum permeability of BHA component (Wb m/A)
Pakistan Engineering Council (PEC), Islamabad, Pakistan 
A Cross-sectional area of ellipse $\left(\mathrm{m}^{2}\right)$

$\theta \quad$ Dip angle

\section{Introduction}

Measurement while drilling tool is a technique used for the collection of surveying data during drilling operations. This tool is commonly used to establish communication between surface and subsurface tools. The mode of transmission of data between the two ends is done through drilling fluid which flows inside the wellbore. For any directional well, the two main parameters are inclination and azimuth. The former is obtained through accelerometer, while the latter is acquired through magnetometer. In general, it is discerned that measurements from magnetometer deteriorate by drill string magnetization, borehole fluid contamination and earth's magnetic field (Priest et al. 2014).

The magnetic field of the earth is primarily produced within its interior and is responsible for providing a shielding effect to the earth. This field is also known as geomagnetic field, which is extended from the earth's outer core to the magnetopause. This field is also considered to be the superposition of magnetic field that is developed by different sources and it changes with time that is affected by the flow of the current in upper atmosphere (Zijsling and Wilson 1989). An error of $50 \mathrm{nT}$ is considered to be the limit beyond which drilling is no longer accurate. It is also essential to minimize these anomalies which are been developed from earth's magnetic field, as they are one of the causes of uncertainty in azimuth (Edvardsen 2015; Mitkus et al. 2019). Besides earth's magnetic field, significant changes in MWD tool measurement occur while drilling in environment containing magnetic strata or localized magnetic geological units. These strata are responsible for creating substantial risk for those directional wells in which MWD tool is used as a surveying tool (Ngugi 2002).

Apart from the geomagnetic field, there is also local magnetic field known as magnetic contamination (Priest et al. 2014) that is a major source of generating magnetic disturbances in MWD sensor performance. In drilling, this local field is associated with drill string, especially the bottomhole assembly (BHA) components, or when the wellbore is nearing an adjacent cased well (Priest et al. 2014). The interferences from the bit and other magnetic components in drill string (DS) affect the tool functionality to a great extent, so to model this magnetic interference, it is common to consider the three orthogonal components acting along each magnetometer axis. Out of three, one is axial component that is acting along the hole and has the dominant effect on azimuth and wellbore position, while the other two are acting in cross-axial and in transverse plane and have minimum impact because of tool-face-dependent nature (Edvardsen
2015; Edvardsen et al. 2013; Ekseth 1998). The axial component of the tool is dependent upon the interferences from the bit and the dip angle that is associated with the magnetic field of the earth (Ekseth 1998).

Another thing that is of great concern is the inclination of a well. The well becomes horizontal as inclination becomes $90^{\circ}$, and when it is greater than $90^{\circ}$, the well starts to moves upward; in that case, the physical distance between the bit and MWD sensor decreases and magnetic interference becomes more dominant which ultimately increases the positional uncertainty in the well (Berchan 2015).

To counter the effect of these two factors, it is extremely crucial to study the behavior of magnetic field before the start of any drilling activity. As for Pakistan, these interferences are quite low, since it is situated in region where the horizontal component of the earth's magnetic field is high. Also, the magnetic anomalies which are developed from the earth's magnetic field are relatively small.

\section{Scope of work}

Drill string is considered as a long cylindrical metallic body, which is utterly responsible for the disturbance in earth's magnetic field. The magnetism in drill string causes distortion in the horizontal component of the earth's magnetic field, which ultimately affects the measurement of magnetic azimuth. The aim of the study is to determine the amount of interferences which is created from drill string components and their impact on MWD sensors. An exemplar BHA is analyzed in this work, and the amount of magnetic susceptibility that each component developed during drilling was investigated.

The study only revolves around the drill string magnetization in two different areas having different latitudes. This magnetization is considered to be the sum of magnetic contributions from numerous steel components in drill string (Grindrod and Wolff 1983). However, it does not incorporate the magnetization of the nearby formation nor the earth's own magnetic strength. The study also includes the sensitivity analyses of magnetization for different lengths of nonmagnetic drill collar (NMDC) in BHA and their impact on magnetometer sensor in MWD. This study carries immense importance as deviation of certain degree from the planned well path can increase the overall cost of the project because of multiple sidetracking. These fluctuations are because of horizontal component of the earth's magnetic field that depends upon the dip angle (Edvardsen et al. 2013). However, the scope of this study lacks the formation and offset data for the two zones, and this limits the works since magnetic ores and casing string in near vicinity can also fluctuate the sensor performance. 


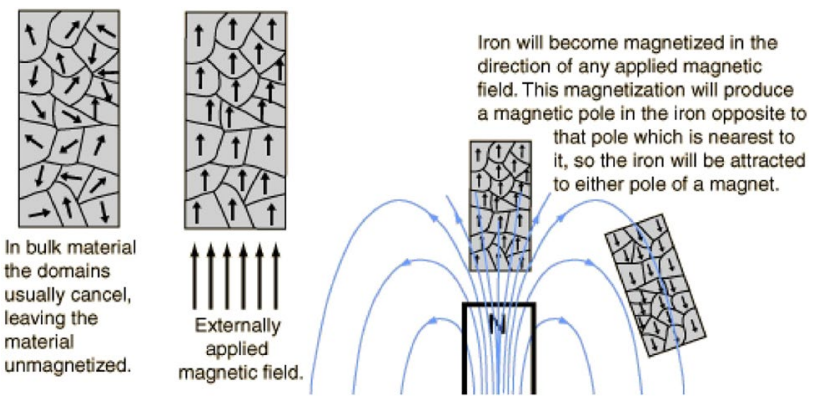

Fig. 1 Effect of magnetic field on magnetic material (Phillips and Aklestad 2015)

\section{Methodology}

Reliability of the final azimuth reduces with an increase in drill string interferences (Edvardsen et al. 2013). To tackle this error source, it is important to ensure that either the physical distance between the sensors and the magnetic components should increase or the length of nonmagnetic drill collar should be appropriate. During the well planning phase, it is often necessary to evaluate these interferences from each BHA component so that appropriate action can be taken. For the estimation of magnetization, drill string is considered as a suspended bar magnets, with each of its components behaving as a tiny magnet or magnetic domain (Phillips and Aklestad 2015).

A magnetic domains is comprised of a group of atomic magnets all pointing in the same direction. If a magnetic material is unmagnetized than the magnetic domains inside that material are not aligned and are randomly arranged as shown in Fig. 1 (Phillips and Aklestad 2015). Similarly, for a magnetic permeable object, magnetic domain will get aligned if it is placed in a magnetic field, which will produce different polarities on either end (Phillips and Aklestad 2015). These poles are responsible for generating a demagnetizing field that is opposite to the direction of applied field and is in charge of decreasing the overall magnetization inside an object (Phillips and Aklestad 2015).

In this study, drill string was divided into different components with each of its constituents being assumed to be a prolate sphere. Magnetization for each element was determined separately using Eq. 1, where $N$ is the demagnetizing factor. Equation 2 shows the dependency of demagnetizing factor on $m$ which is the ratio of semi-major axis to the semi-minor axis of an ellipsoid (Osborn 1945; Phillips and Aklestad 2015):

$B_{\text {in }}=\mu_{o}\left(\frac{H_{a d}}{N}\right)+\mu_{o}\left(\frac{N-1}{N}\right) H_{\text {in }}$
$N=\left(\frac{1}{m^{2}-1}\right)\left[\frac{m}{2 \sqrt{m^{2}-1}} \ln \left(\frac{m+\sqrt{m^{2}-1}}{m-\sqrt{m^{2}+1}}\right)-1\right]$

Since components of drill string are made up of steel which is a ferromagnetic material, the determination of demagnetizing factor is extremely significant. The knowledge related to this factor is equivalent to the corresponding demagnetizing fields (Beleggia et al. 2006). This field is non-uniform both inside and outside of a body, except for the case when a body is an ellipsoid (Beleggia et al. 2006); this is because of the uniform distribution of the charges on either end of an ellipsoid, which results in the total charge equivalent to zero (Phillips and Aklestad 2015). As the charges are concentrated on either end, it results in magnetic dipole of a particular strength which can be calculated using Eq. 3 (Phillips and Aklestad 2015):

$Q=\frac{\mu_{o}\left(\mu_{\max }-1\right) H_{a d} A}{\left(1+N\left(\mu_{\max }-1\right)\right)}$.

As constituents of bottom-hole assembly (BHA) are all connected together, it results in contiguous region of magnetic field having magnetic dipole on either end of its components that can be obtained using Eq. 3 (Phillips and Aklestad 2015). This strength is then transformed into the interferences from either end using Eq. 4 (Phillips and Aklestad 2015):

$B_{\text {interference }}=\frac{-Q}{4 \pi r^{2}}$.

In total, 26 error sensor sources are associated with MWD tool (Jamieson 2009). The output of Eq. 4 is then used to formulate the impact of BHA interference on magnetometer, especially the biased terms. ${ }^{1}$ Three-dimensional survey information is gathered from three axes of measurement in magnetometer as shown in Fig. 2. The $Z$-axis component bias error is mostly affected by the interferences from the BHA. This is an axial component that is acting along the hole and has the dominant effect on azimuth and wellbore position, while the other two are acting in cross-axial and in transverse plane and have minimum impact because of toolface-dependent nature. Equation 5 represents the azimuth for the magnetic surveying, where $b_{x}, b_{y}$ and $b_{z}$ are three magnetometer orthogonal measurements as shown in Fig. 2. The major uncertainty in the azimuth is caused by the parameter $b_{z}$ (Edvardsen et al. 2013), which can be observed if Eq. 5 is differentiated with respect to $b_{z}$; the result is shown in Eq. 6 . The factor $\sin I \sin A_{m} / B \cos \theta$ takes into the account both ${ }_{1}$ Error sources that have nonzero mean and there ellipses of uncer-
tainty are offset.

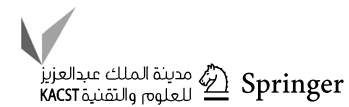




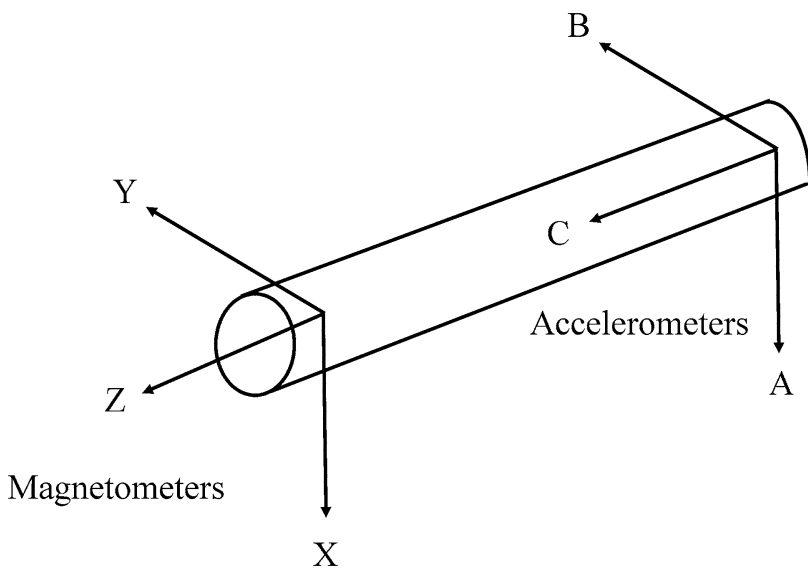

Fig. 2 Measurement while drilling sensor and it axes

the geomagnetic position and the well trajectory (Edvardsen et al. 2013; Jamieson 2009):

$A=\delta=\arctan \frac{b_{x} \cos \tau-b_{y} \sin \tau}{\cos I\left(b_{x} \sin \tau+b_{y} \cos \tau\right)+b_{z} \sin I}$

$\Delta A=-\frac{\sin I \sin A_{m}}{B \cos \theta} \Delta b_{z}$

where $B$ is the magnetic field intensity for the particular location and $\theta$ is the dip angle. Together, $B \cos \theta$ makes the horizontal component of the earth's magnetic field. At high latitude, especially in Barents and North Sea, this factor of the earth's magnetic field is reasonably small; therefore, the uncertainty associated with azimuth $\Delta A$ is large enough to create problems in wellbore positioning (Edvardsen et al. 2013). However, for Pakistan, the magnetic anomalies that are present in earth's atmosphere are fairly inadequate to give rise to any uncertainty in azimuth. Equations 7 and 8 define the remaining two-axis magnetometer bias error terms for MWD tool; these terms are independent of DSI and are only the function of geomagnetic location (Jamieson 2009):

$\Delta A_{x}=-\frac{\cos I \sin A_{m}}{B \cos \theta}$

$\Delta A_{y}=\frac{\cos A_{m}}{B \cos \theta}$.

\section{Results and discussion}

The study involves around two wells having different geomagnetic locations and latitudes. The first well is in the region of Barents Sea having latitude $73^{\circ} 19^{\prime}$ and longitude $37^{\circ} 15^{\prime}$, whereas the other well is the region of Pakistan having longitude $69^{\circ} 04^{\prime}$ and latitude $27^{\circ} 77^{\prime}$. An exemplar BHA is considered, in which sensors were placed $25.65 \mathrm{~m}$ away from the bit. The study was carried out in 2017, in which a total of 38 survey stations were evaluated. During this study, the magnetic field strength for the year 2017 was determined using the WMM model. ${ }^{2}$ Figure 3 shows detailed analysis of the total magnetic field for the two regions, of which one is located in Barents Sea, whereas the other is situated in the region of Pakistan near Sukkur.

The BHA in this study was subdivided into three main regions. Region 1 was allotted the length from the bit to the stabilizer with the total magnetic length of $12.35 \mathrm{~m}$. This region is $13.30 \mathrm{~m}$ away from D\&I sensors. Region 2 comprises the distance from stabilizer to the Universal Bottom Hole Orientation $\mathrm{Subs}^{3}$ (UBHO), both having magnetic capabilities that deteriorate the performance of sensors. This region is $3 \mathrm{~m}$ in length and is most contiguous to the sensors; however, this area does not show any interference as both the lower and upper ends of the region have the same magnitude but different polarities. Region 3 is comprised of crossover and collars with a total magnetic length of $74.7 \mathrm{~m}$.

Region 1 shows a relatively high interference of about $140.14 \mathrm{nT}$. To reduce this interference from the bit, selection of the appropriate length of nonmagnetic drill collar (NMDC) is of utmost concern. In the above BHA example, the size of NMDC is $9.5 \mathrm{~m}$ that is responsible for this $140.14 \mathrm{nT}$ of interference. A sensitivity analysis, indicated in Fig. 4, has been performed related to the proper selection of NMDC, which shows that as the size of NMDC is increased, there is a drastic reduction in the interference, which is an indication that the sensors are moving away from the potential interference source. It was further found out that in Pakistan these bit interferences were 15\% lower of what they were in Barents Sea. A comparative investigation was further performed as shown in Fig. 5, that specifies the problem of not using the recommended length of NMDC and the possible azimuth error that will produced in the measurements.

For the true azimuth, the error should be below the $0.25^{\circ}$ (Grindrod and Wolff 1983). BHA used in this study is comprised of $9.5 \mathrm{~m}$ of NMDC which is utterly responsible for the distortion in Z-component of magnetometer. It is observed that if the difference between the actual length and recommended length is significant, then the azimuthal error is substantially high. The arrow shows that the error is getting smaller when the difference between actual and

\footnotetext{
${ }^{2}$ A standard model for monitoring the Earth's magnetic field along with the attitude.

${ }^{3}$ A float valve controls the fluid flow at the bottom of drill string.
} 
Fig. 3 Total magnetic field comparison between a region in Pakistan and Barents Sea

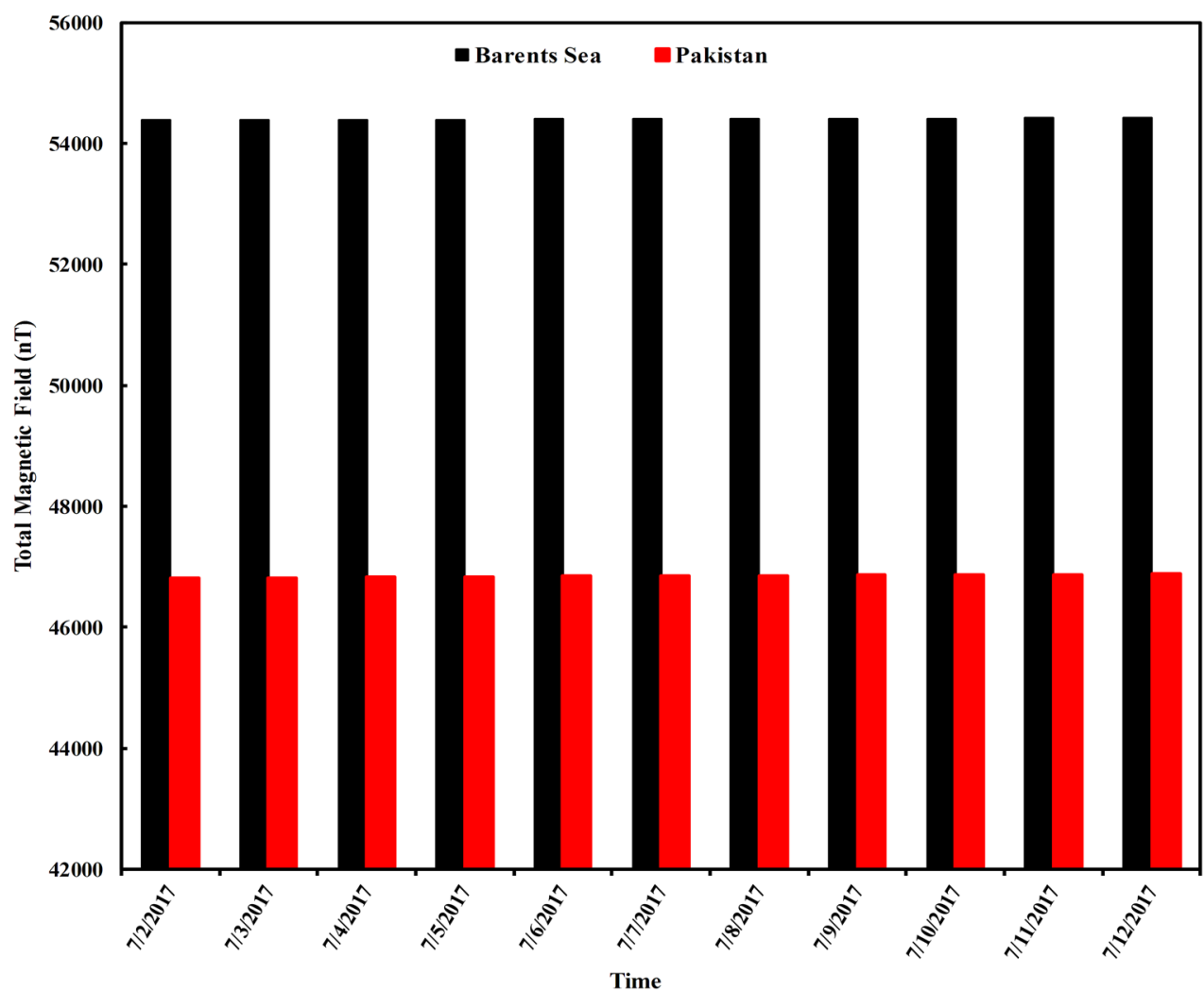

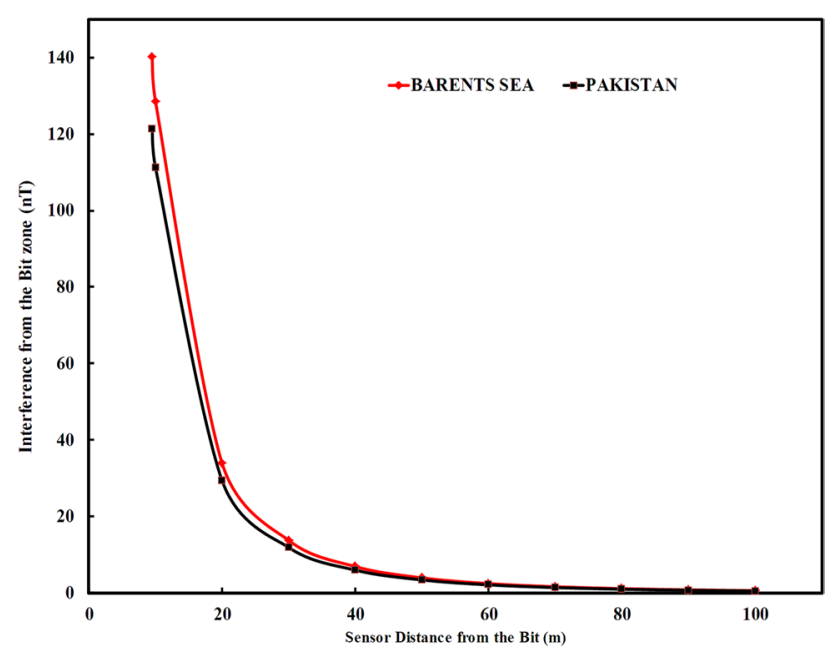

Fig. 4 Bit interferences from different NMDC lengths

recommended lengths is getting closer to each other (Grindrod and Wolff 1983).

Since the drill string is in continuous rotation, it causes the magnetization to get aligned along the drill string axis (Jamieson 2009). It is also observed that the $Z$-component of the magnetometer biased (MB) is the most affected component. This parameter not only depends upon the total magnetic field (TMF), but also affected by bit interference as shown in Eq. 6. Figure 6 shows the impact of bit interference on $Z$-component of magnetometer in Barents Sea. It is monitored that as the size of NMDC increases, the error associated with this components starts to decrease and moves toward zero. In the analysis, if the size of NMDC is $9.5 \mathrm{~m}$, then it shows a high degree of fluctuations in this parameter; however, if the size of NMDC increases, it is observed that the error term starts to reduce significantly and moves toward zero. This is possible because of an increase in physical distance between the MWD sensors and the bit, which enables the sensor to perform with high degree of accuracy. However, the cost of increasing the size of NMDC should be taken into consideration, as increasing the size needs some additional equipment that ultimately affects the overall cost of BHA. Nevertheless, this cost can be managed in comparison with the problem accompanied in well positioning surveys that result in multiple sidetracking.

Equation 6 further shows that the uncertainty in azimuth is also dependent upon the factor of $B \cos \theta$, which is also known as the horizontal component of earth's magnetic field (Edvardsen et al. 2013). In regions where magnetic latitudes are high, this factor is quite small which corresponds to the large magnetic azimuthal error. This is also shown in Fig. 7, where the same well is considered in a region of Pakistan having lower magnetic latitude, which results in lower azimuth error in Z-component. Both results were obtained by using $9.5 \mathrm{~m}$ of NMDC in the BHA. 
Fig. 5 Possible error in azimuth for not using the recommended length of NMDC
Fig. 6 Impact of NMDC length on $Z$-axis component of magnetometer for Barents Sea
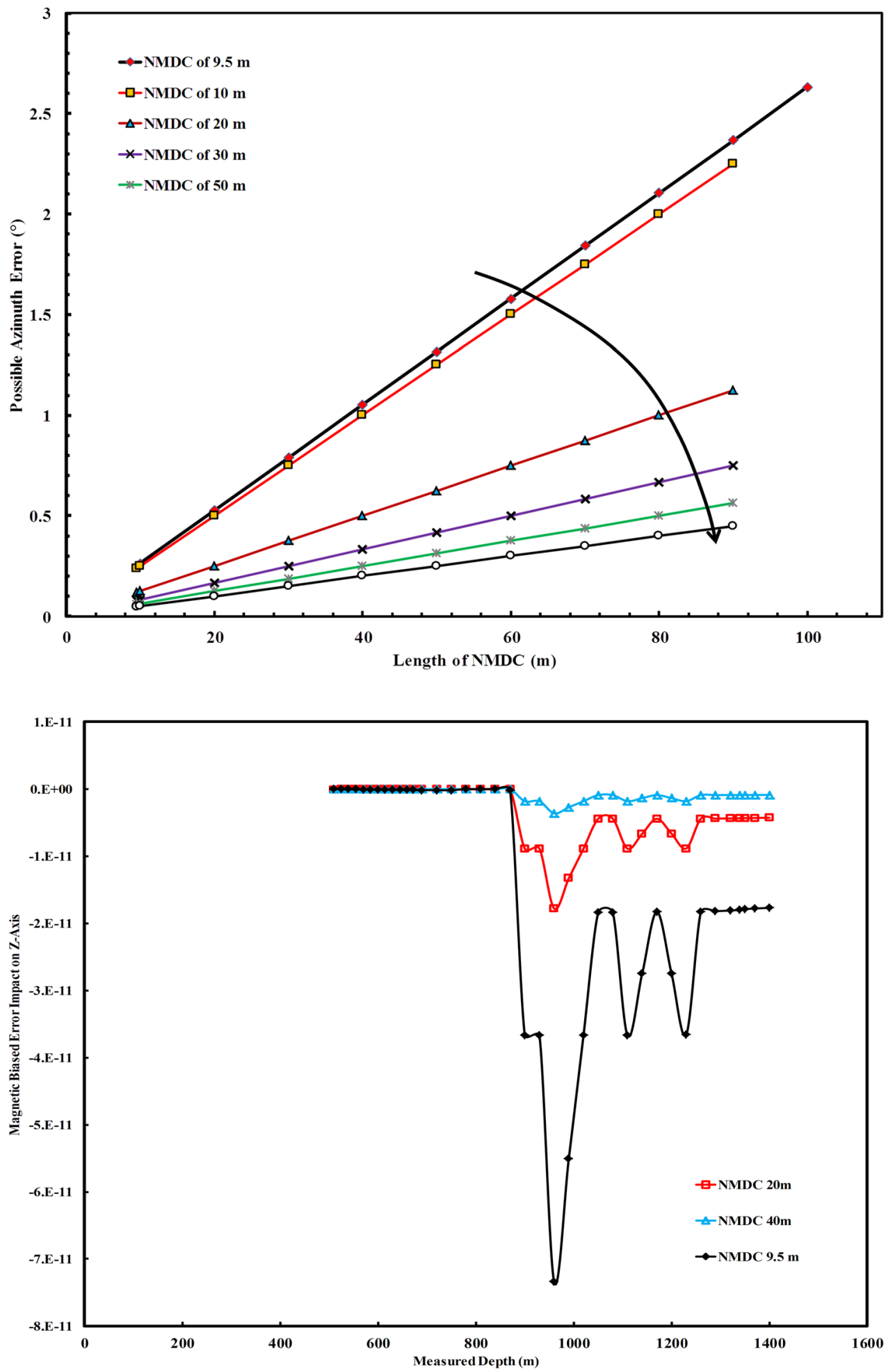

Figures 8 and 9 show the error that is observed in $X$ and $Y$-components of magnetometer. It was observed that the $X$-component shows some fluctuations throughout the year in azimuth. However, the $Y$-component remains constant even though the field changes from 54,381.3 to $54,421.6 \mathrm{nT}$. This indicates that the $Y$-component is independent of the magnetic field of the earth. On the contrary, both of these components are independent of the bit interferences. In the region of Pakistan, the $X$-component azimuthal error was decreased by $73 \%$ as the TMF of this region is quite low since it is away from the magnetic poles. 
Fig. 7 Comparison between two different regions' magnetometer biased errors

Fig. 8 Error in magnetometer $X$-component for Barents Sea

\section{Conclusion}

It was concluded from the study that:

1. Some of the error sources have considerable impact on the total uncertainty due to the north dominance trajectory.

2. Bit is made up of carbon steel having a high magnetic permeability and profound magnetic characteristics.
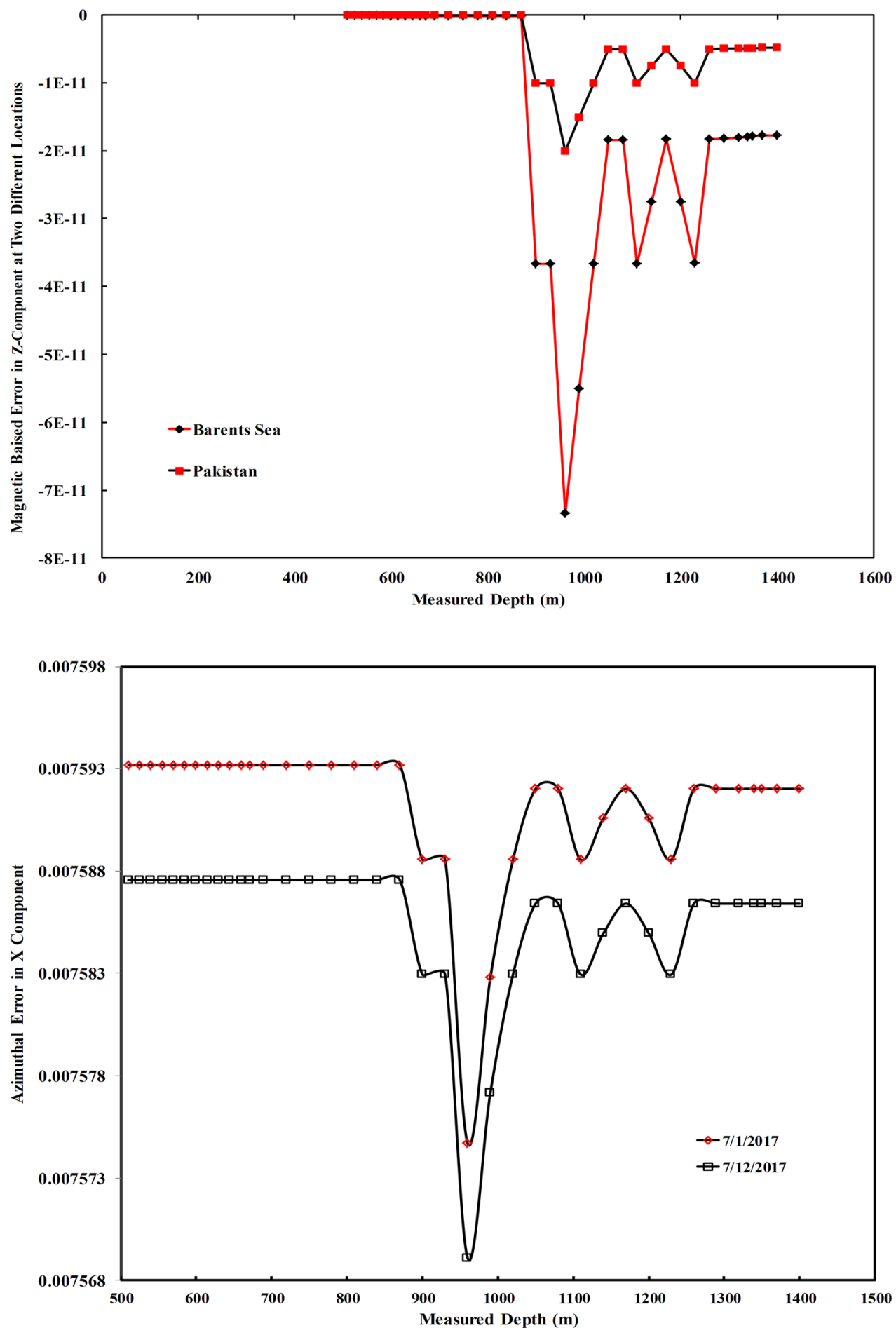

This region is considered to be the highest interference region in the entire $\mathrm{BHA}$, which can be reduced by increasing the size of NMDC.

3. The Z-component of the magnetic biased is assumed to be the most effected component by bit interference. This impact can again be reduced by increasing the size of NMDC. The optimal distance based on the study is found to be $40 \mathrm{~m}$ for the Barents Sea, as it causes an interfer- 
Fig. 9 Error in magnetometer $Y$-component for Barents Sea

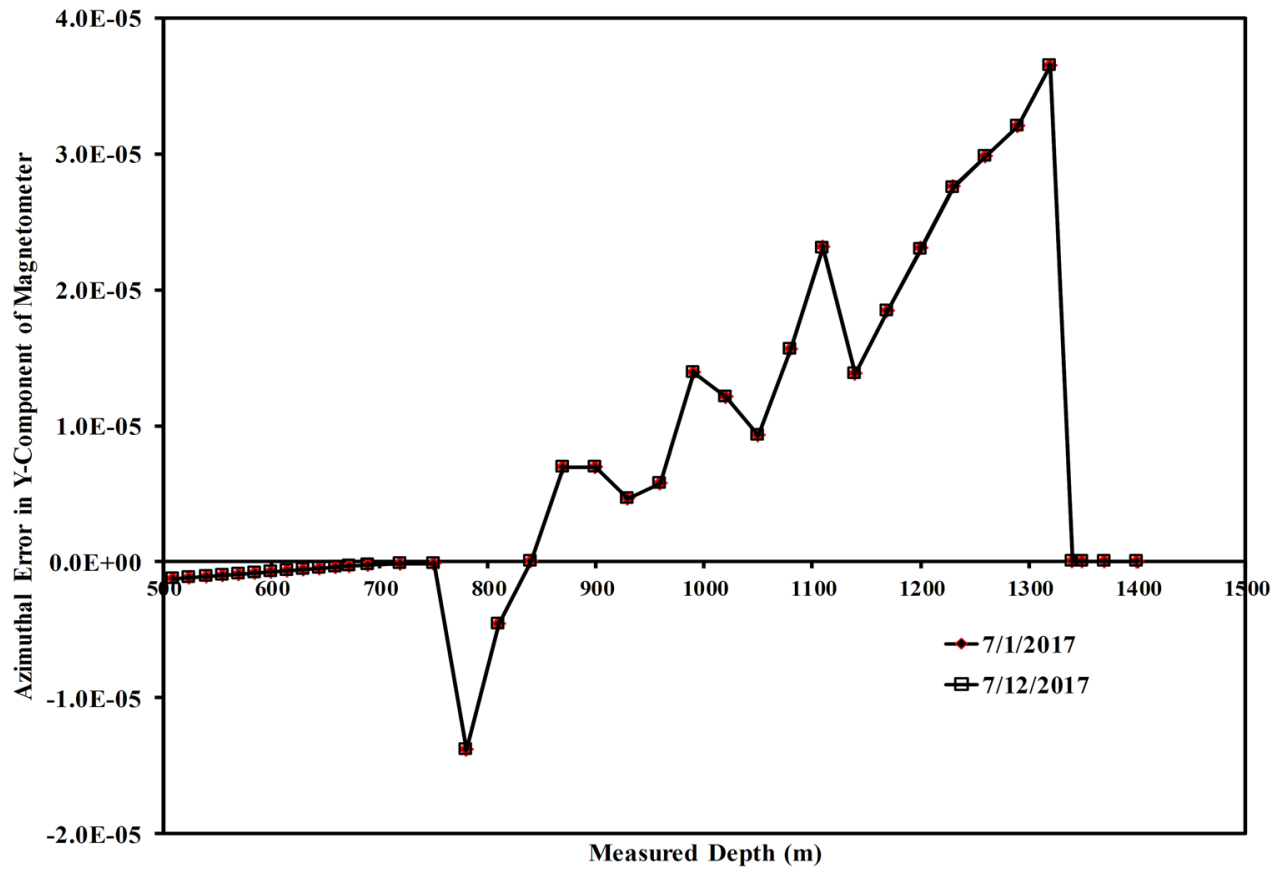

ence of $6.92 \mathrm{nT}$ that is manageable. However, for Pakistan $9.5 \mathrm{~m}$ of NMDC was producing $5.62 \mathrm{nT}$ interference.

4. The weighting function in $Z$-component which quantifies as $\sin I \sin A_{m} / B \cos \theta$ accounts for uncertainty in azimuth with respect to geomagnetic location and latitude.

5. At the region where magnetic latitudes are high, the horizontal component of the earth's magnetic field is quite small, which results in high azimuthal error. This is also justified while comparing the $Z$-component error in the region of Pakistan and Barents Sea.

6. $X$-component of the magnetometer is unaffected by bit interference; however, it shows some dependency on geomagnetic field. On the other hand, the $Y$-component is independent on geomagnetic location and latitude.

Open Access This article is distributed under the terms of the Creative Commons Attribution 4.0 International License (http://creativeco mmons.org/licenses/by/4.0/), which permits unrestricted use, distribution, and reproduction in any medium, provided you give appropriate credit to the original author(s) and the source, provide a link to the Creative Commons license, and indicate if changes were made.

\section{References}

Beleggia M, De Graef M, Millev Y (2006) Demagnetization factors of the general ellipsoid: an alternative to the Maxwell approach. Phil Mag 86(16):2451-2466

Berchan BA (2015) Drilling, completion, intervention and P\&A design and operations. NTNU, Trondheim

Edvardsen I (2015) Effects of geomagnetic disturbances on offshore magnetic directional wellbore positioning in the Northern Auroral Zone. The Arctic University of Norway, Tromso
Edvardsen I, Nyrnes E, Johnsen MG, Hansen TL (2013) Improving the accuracy and reliability of MWD/magnetic-wellbore-directional surveying in the Barents sea. In: Annual technical conference and exhibition 2013. SPE, New Orleans

Ekseth R (1998) Uncertainties in connection with the determination of wellbore positions. Norwegian University of Science and Technology, Trondheim

Grindrod SJ, Wolff JM (1983) Calculation of NMDC length required for various latitudes developed from field measurements of drill string magnetization. In: IADC/SPE drilling conference. SPE, New Orleans

Jamieson PA (2009) Introduction to wellbore positioning. University of the Highlands and Islands, Inverness

Mitkus A, Maus S, Willerth M, Reetz A (2019) Challenges and solution for accurate wellbore placement in the Barents sea. In: SPE/ IADC international drilling conference and exhibition. 2019. SPE, The Hague

Ngugi PK (2002) Technical, economic and institutional evaluation of adopting directional drilling by Kengen. The United Nations University, Shibuya City

Osborn JA (1945) Demagnetization factors of the general ellipsoid. Phys Rev 67(11):351-357

Phillips W, Aklestad D (2015) Modeling drill string magnetic interference of wellbore surveys using demagnetizing factors. In: SPE/IADC drilling conference and exhibition SPE, Editor. SPE, London

Priest J, Quinn T, Frost Jr E (2014) Magnetic interference effects on accelerometer and magnetometer data: detection, quality control and correction. In: SPWLA 55th annual logging symposium. 2014. Society of Petrophysicists and Well Log Analysts (SPWLA), Abu Dhabi

Zijsling DH, Wilson RA (1989) Improved magnetic surveying techniques: field experience. In: Offshore Europe 89. 1989. SPE, Aberdeen

Publisher's Note Springer Nature remains neutral with regard to jurisdictional claims in published maps and institutional affiliations. 